\title{
Functional Gastrointestinal Disorders in Young Military Men
}

\author{
Chang Seok Bang*, Yeon Soo Kim*, Jin Hyung Han ${ }^{\dagger}$, Yong Sub Lee*, Gwang Ho Baik*, Jin Bong Kim*, Ki Tae Suk*, Jai \\ Hoon Yoon*, and Dong Joon Kim* \\ ${ }^{*}$ Department of Internal Medicine, Hallym University College of Medicine, Chuncheon, and ${ }^{\dagger}$ Department of Internal Medicine, Dankook \\ University College of Medicine, Cheonan, Korea
}

Background/Aims: To estimate the prevalence and evaluate the associated psychological factors of functional gastrointestinal disorders (FGIDs) in males in their twenties who are currently enrolled in military service. Methods: A total of 1,073 men in the Korean army were asked to complete questionnaires based on the Rome III criteria and Symptom Checklist-90-revised (SCL-90R). The prevalence of FGIDs was estimated, and the associated psychological factors were evaluated. Results: A total of 967 men participated. The total prevalence of FGIDs was 18.5\% (age-adjusted prevalence, 18.1\%; 95\% confidence interval [Cl], $15.3 \%$ to $20.8 \%$ ). The total SCL-90R scores were higher in men with FGIDs than men without FGIDs (24 [interquartile range, 13 to 44 ] vs 13 [5 to 28], p<0.001) and higher in men with overlapping syndromes than in those with single FGIDs (31 [18 to 57] vs 14 [5.75 to 29], $p<0.001$ ). Somatization (odds ratio [OR], $1.141 ; 95 \% \mathrm{Cl}, 1.09$ to $1.20 ; p<0.001)$, obsessive-compulsive behaviors (OR, 1.084; 95\% $\mathrm{Cl}, 1.03$ to 1.14; $\mathrm{p}=0.002$ ) and depression (OR, 0.943; 95\% Cl, 0.90 to 0.99; $p=0.020$ ) were identified as independent predictive factors for FGIDs. Conclusions: FGIDs are common among men in their twenties who are fulfilling their military duty. Somatization and obsessive-compulsive features from the tense atmosphere are associated with the development or progression of FGIDs. Patients who exhibit overlapping syndromes require greater attention given their more severe psychopathology. (Gut Liver 2015;9:509-515)

Key Words: Gastrointestinal diseases; Military; Psychologic factors

\section{INTRODUCTION}

Functional gastrointestinal disorders (FGIDs) are commonly observed in clinical practice. ${ }^{1}$ Patients complain a variety of symptoms, as these disorders result in a great burden in terms of quality of life and health care expenditures. ${ }^{2}$ The Rome criteria, which are based on symptom clusters, are currently used to diagnose FGIDs. ${ }^{3}$ This metric was updated in 2006, as the Rome III version, and 28 disorders are included in these criteria. ${ }^{4}$ There have been studies estimating the prevalence of FGIDs using Rome III criteria in Korea;, ${ }^{5,6}$ however, the exact prevalence and pathophysiology of FGIDs remain unclear.

Young soldiers, who are highly trained and submitted to strictly imposed discipline, tend to face more serious stress than the general population. All Korean males in their twenties who do not have a serious disability have an obligation to fulfill a term of military service. However, studies on FGIDs investigating this population are lacking.

The aim of this study was to estimate the prevalence and evaluate the associated psychological factors of FGIDs among males in their twenties currently completing military service.

\section{MATERIALS AND METHODS}

\section{Ethics statement}

This study was conducted according to the principles expressed in the Declaration of Helsinki. Voluntary participation was requested, and written informed consent was obtained from each participant. This study was approved by the Institutional Review Board of the Armed Forces Medical Command of Korea before initiation of this study (AFMC-13-IRB-010).

\footnotetext{
Correspondence to: Gwang Ho Baik

Department of Internal Medicine, Hallym University Chuncheon Sacred Heart Hospital, Hallym University College of Medicine, 77 Sakju-ro, Chuncheon 200-704, Korea

Tel: +82-33-240-5821, Fax: +82-33-241-8064, E-mail: baikgh@hallym.or.kr

Received on March 26, 2014. Revised on May 1, 2014. Accepted on May 4, 2014. Published online December 5, 2014

pISSN 1976-2283 eISSN 2005-1212 http://dx.doi.org/10.5009/gnl14109

Chang Seok Bang and Yeon Soo Kim contributed equally to this work as first authors.

() This is an Open Access article distributed under the terms of the Creative Commons Attribution Non-Commercial License (http://creativecommons.org/licenses/by-nc/4.0) which permits unrestricted non-commercial use, distribution, and reproduction in any medium, provided the original work is properly cited.
} 


\section{Patients and methods}

Between January and May 2013, a total of 1,073 participants from eight different regiments of three different divisions of Republic of Korea Army were asked to complete questionnaires based on the Rome III criteria and Symptom Checklist90-revised (SCL-90R). The three divisions were located in the Gangwon, Gyeonggi, and Chungcheongnam provinces of Korea, respectively. The study population included male soldiers in their twenties who participated voluntarily. The survey was performed face-to-face in the military at doctors' offices by three military doctors (C.S.B., J.H.H., and Y.S.L.). The prevalence of FGIDs was estimated, and all associated psychological factors were evaluated using the Rome III criteria and a SCL-90R based questionnaire. Participants who were positive for alarm symptoms during the screening for organic diseases or who presented an incomplete questionnaire were excluded from this study.

\section{Questionnaires}

This study was conducted using a self-reported questionnaire. The Korean version of a Rome III criteria-based questionnaire was produced according to the following steps: (1) independent translations of the original version of the Rome III criteria by two native speakers of Korean who are fluent in English; (2) comparison of the two versions of the questionnaire with the two translators making adjustments for better understanding to create a reconciled version; (3) evaluation of the questionnaire by three internal medicine doctors, followed by revision until interobserver agreement was achieved and its use was approved in soldiers; and (4) administration of a pretest to 300 soldiers in three different regiments to detect unrecognized errors, which were corrected in the final version. The questionnaire consisted of 86 queries, including 75 Rome criteria-based questions, seven alarming symptom questions, and four demographic information questions. Alarm symptoms included unintentional weight loss, blood in the stool, a history of organic bowel disease or intestinal surgery, unexplained anemia or fever, and awakening due to abdominal pain during the night time.

The SCL-90 is a questionnaire that was developed in 1973 to assess psychological distress for clinical practice and research purposes. ${ }^{7}$ The Korean version of the SCL-90R was created and validated in $1984{ }^{8}$ This 5-point Likert-scale questionnaire consists of 90 queries, and each question represents a single psychological symptom. Using this questionnaire, the psychological symptoms can be classified into nine categories (somatization, obsessive-compulsive, interpersonal sensitivity, depression, anxiety, hostility, phobic anxiety, paranoid ideation, and psychoticism), and the global severity can be assessed. The scores of global severity and of each category are expressed as t scores. ${ }^{89}$

\section{Sample size}

Based on a study of Chinese college and university students, ${ }^{10}$ representing the same age groups that are in our study, a sample size was calculated. According to that study, the prevalence of FGIDs among males was estimated at 20.4\%. The authors assumed that the total prevalence of FGIDs in the military would be $25 \%$; thus, the sample size was calculated with $80 \%$ power at a two-sided p-value of 0.05 and a drop rate of 10\%. A minimum sample size of 700 was required.

\section{Statistical analysis}

Continuous data are expressed as the medians and the interquartile ranges, and categorical data are given as the frequencies (percentages). The Mann-Whitney test and Fisher exact test were used to evaluate the baseline characteristics and to compare the SCL-90R scores (soldiers with FGIDs vs without FGIDs / soldiers with overlap syndrome vs single FGIDs), because of the nonnormal distribution of the data. Age-adjusted prevalence of FGIDs was calculated with the population in 2010 Korean Census as the standard population (classified and weighted by 5-year age interval). A multivariate logistic regression test was used to detect the independent psychological risk factors associated with FGIDs. A p-value $<0.05$ was considered statistically significant for all tests. The analysis was performed using the SPSS software version 18.0 (SPSS Inc., Chicago, IL, USA).

\section{RESULTS}

\section{The characteristic of enrolled population}

Of the 1,073 soldiers who took the questionnaire, 106 men

Table 1. Clinical Characteristics of Total Enrolled Population

\begin{tabular}{lcccc}
\hline \multicolumn{1}{c}{ Characteristic } & Total $(\mathrm{n}=967)$ & Soldiers with FGIDs $(\mathrm{n}=179,18.5 \%)$ & Soldiers without FGIDs $(\mathrm{n}=788,81.5 \%)$ & $\mathrm{p}$-value \\
\hline Age, yr & $21(20-21)$ & $21(20-22)$ & $21(20-21)$ & 0.01 \\
BMI & $22.2(20.8-24.0)$ & $22.4(20.8-24.6)$ & $22.2(20.8-23.9)$ & 0.43 \\
Smoking & $478(49.4)$ & $94(52.5)$ & $384(48.7)$ & 0.36 \\
$\quad$ Cigarette per day & $0(0-10)$ & $3(0-10)$ & $0(0-10)$ & 0.24 \\
Alcohol & $698(72.2)$ & $135(75.4)$ & $563(71.4)$ & 0.31 \\
SCL-90R & $15(6-30)$ & $24(13-44)$ & $13(5-28)$ & $<0.001$ \\
\hline
\end{tabular}

Data are presented as median (interquartile range) or number (\%).

FGIDs, functional gastrointestinal disorders; BMI, body mass index; SCL-90R, Symptom Checklist-90-revised. 
Table 2. Age-Adjusted Prevalence of Functional Gastrointestinal Disorders

\begin{tabular}{ccccc}
\hline Age class, yr & Total cases of each age class & Cases with FGIDs & Prevalence of FGIDs, \% & Standard population of Korean Census \\
\hline $15-19$ & 100 & 17 & 17 & $1,826,179$ \\
$20-24$ & 850 & 156 & 18.4 & $1,625,371$ \\
$25-29$ & 16 & 6 & 37.5 & $1,802,805$ \\
$30-35$ & 1 & 0 & 0 & $1,866,397$ \\
Total & 967 & 179 & 18.5 & - \\
& & Age-adjusted prevalence of FIGDs: $18.1 \%(95 \%$ CI, 15.3\%-20.8\%)
\end{tabular}

FGIDs, functional gastrointestinal disorders; CI, confidence interval.

Table 3. Proportion of Functional Gastrointestinal Disorders

\begin{tabular}{lcc}
\hline \multicolumn{1}{c}{ FGIDs } & $\begin{array}{c}\text { No. (\% of all } \\
\text { population) }\end{array}$ & \% of FGIDs \\
\hline Functional dyspepsia & $79(8.2)$ & 44.1 \\
Functional chest pain & $49(5.1)$ & 27.4 \\
Functional heartburn & $27(2.8)$ & 15.1 \\
Irritable bowel syndrome & $25(2.6)$ & 14.0 \\
Functional dysphagia & $25(2.6)$ & 14.0 \\
Functional diarrhea & $20(2.1)$ & 11.2 \\
Functional constipation & $13(1.3)$ & 7.3 \\
Globus & $12(1.2)$ & 6.7 \\
Functional bloating & $10(1.0)$ & 5.6 \\
Proctalgia fugax & $5(0.5)$ & 2.8 \\
Chronic idiopathic nausea & $4(0.4)$ & 2.2 \\
Aerophagia & $3(0.3)$ & 1.7 \\
Cyclic vomiting syndrome & $3(0.3)$ & 1.7 \\
Rumination syndrome & $2(0.2)$ & 1.1 \\
\hline
\end{tabular}

FGIDs, functional gastrointestinal disorders.

submitted incomplete questionnaires and were excluded from this study. A total of 967 men participated, for a response rate of $90.1 \%$. The median age was 21 years (interquartile range, 20 to 21 years), and the proportions of smokers and alcohol drinkers were $49.4 \%$ and $72.2 \%$, respectively. The median body mass index (BMI) was 22.2 (interquartile range, 20.8 to 24). The characteristics of the total enrolled soldiers are listed in Table 1.

\section{The prevalence and spectrum of FGIDs}

The total prevalence of FGIDs was 18.5\% $(n=179)$. The ageadjusted prevalence of FGIDs was 18.05\% (95\% confidence interval [CI], 15.3\% to 20.8\%) (Table 2). The proportions of each FGID were as follows: functional dyspepsia (FD) (8.2\%), functional chest pain (FCP) (5.1\%), functional heartburn (FH) (2.8\%), irritable bowel syndrome (IBS) (2.6\%), and functional dysphagia (2.6\%). The prevalence of each FGID in soldiers is shown in Table 3.

Among soldiers with functional dyspepsia, a subtype of postprandial distress syndrome (PDS) was more prevalent than epigastric pain syndrome (EPS) ( $n=78$ vs 1). In the subtype of
Table 4. Subtypes of Functional Dyspepsia and Irritable Bowel Syndrome

\begin{tabular}{lcc}
\hline \multicolumn{1}{c}{ FGIDs } & $\begin{array}{c}\text { No. (\% of all } \\
\text { population) }\end{array}$ & \% of FGIDs \\
\hline Functional dyspepsia & $79(8.2)$ & 44.1 \\
Postprandial distress syndrome & $78(8.1)$ & 43.6 \\
Epigastric pain syndrome & $1(0.1)$ & 0.6 \\
IBS & $25(2.6)$ & 14.0 \\
IBS-C & $3(0.3)$ & 1.7 \\
IBS-D & $10(1.0)$ & 5.6 \\
IBS-M & $9(0.9)$ & 5.0 \\
IBS-U & $3(0.3)$ & 1.7 \\
\hline
\end{tabular}

FGIDs, functional gastrointestinal disorders; IBS, irritable bowel syndrome; IBS-C, constipation type IBS; IBS-D, diarrhea type IBS; IBS$\mathrm{M}$, mixed type IBS; IBS-U, unsubtyped IBS.

Table 5. Types of Overlapping Syndromes

\begin{tabular}{lcc}
\hline Overlap syndrome & No. (\% of all population) & \% of FGIDs \\
\hline Total & $53(5.5)$ & 29.6 \\
FD+FH+FCP & $7(0.7)$ & 3.9 \\
FD+FCP & $6(0.6)$ & 3.3 \\
FD+IBS & $5(0.5)$ & 2.8 \\
IBS+FCP & $4(0.4)$ & 2.2 \\
FH+FCP & $4(0.4)$ & 2.2 \\
FD+IBS+FCP & $4(0.4)$ & 2.2 \\
FD+FH & $2(0.2)$ & 1.1 \\
IBS+FH & $2(0.2)$ & 1.1 \\
\hline
\end{tabular}

Nineteen single combinations of overlapping syndrome were omitted in this table.

FGIDs, functional gastrointestinal disorders; FD, functional dyspepsia; FH, functional heartburn; FCP, functional chest pain; IBS, irritable bowel syndrome.

IBS, diarrhea-type IBS was most prevalent $(n=10)$, followed by mixed $(n=9)$, constipation type $(n=3)$, and unsubtyped IBS $(n=3)$ (Table 4).

\section{Overlap syndrome}

Among the 179 soldiers with FGIDs, 53 soldiers (29.6\%) had combined FGIDs, that is, meeting the criteria for more than one 
Table 6. SCL-90R Scores: Soldiers with Functional Gastrointestinal Disorders versus without Functional Gastrointestinal Disorders

\begin{tabular}{lccc}
\hline $\begin{array}{c}\text { Psychopathologic } \\
\text { variable }\end{array}$ & $\begin{array}{c}\text { Soldiers } \\
\text { with FGIDs } \\
(\mathrm{n}=179,18.5 \%)\end{array}$ & $\begin{array}{c}\text { Soldiers } \\
\text { without FGIDs } \\
(\mathrm{n}=788,81.5 \%)\end{array}$ & $\mathrm{p}$-value \\
\hline Somatization & $4(2-8)$ & $2(1-4)$ & $<0.001$ \\
Obsessive compulsive & $5(2-9)$ & $3(1-7)$ & $<0.001$ \\
Interpersonal sensitivity & $3(1-6)$ & $2(0-4)$ & $<0.001$ \\
Depression & $3(1-8)$ & $2(0-5)$ & $<0.001$ \\
Anxiety & $2(1-3)$ & $1(0-2)$ & $<0.001$ \\
Hostility & $1(1-3)$ & $0(0-1.75)$ & $<0.001$ \\
Phobic anxiety & $0(0-1)$ & $0(0-0)$ & $<0.001$ \\
Paranoid ideation & $1(0-2)$ & $0(0-1)$ & $<0.001$ \\
Psychoticism & $0(0-2)$ & $0(0-1)$ & $<0.001$ \\
SCL-90R & $24(13-44)$ & $13(5-28)$ & $<0.001$ \\
\hline
\end{tabular}

Data are presented as median (interquartile range).

SCL-90R, Symptom Checklist-90-revised; FGIDs, functional gastrointestinal disorders.
Table 7. SCL-90R Scores: Soldiers with Overlap Syndrome versus Single Functional Gastrointestinal Disorders

\begin{tabular}{lccr}
\hline \multicolumn{1}{c}{$\begin{array}{c}\text { Psychopathologic } \\
\text { variable }\end{array}$} & $\begin{array}{c}\text { Combined } \\
\text { FGIDs } \\
(\mathrm{n}=53,5.5 \%)\end{array}$ & $\begin{array}{c}\text { Single } \\
\text { FGIDs } \\
(\mathrm{n}=126,13 \%)\end{array}$ & p-value \\
\hline Somatization & $6(3-9)$ & $2(1-5)$ & $<0.001$ \\
Obsessive compulsive & $7(3-10.5)$ & $3(1-7)$ & $<0.001$ \\
Interpersonal sensitivity & $4(2-8)$ & $2(0-5)$ & $<0.001$ \\
Depression & $4(1.5-9)$ & $2(0-6)$ & 0.001 \\
Anxiety & $2(1-4)$ & $1(0-2)$ & $<0.001$ \\
Hostility & $1(0-3)$ & $0(0-2)$ & $<0.001$ \\
Phobic anxiety & $0(0-1)$ & $0(0-0)$ & 0.006 \\
Paranoid ideation & $2(0-5)$ & $0(0-1)$ & $<0.001$ \\
Psychoticism & $1(0-3)$ & $0(0-1)$ & 0.001 \\
SCL-90R & $31(18-57)$ & $14(5.75-29)$ & $<0.001$ \\
\hline
\end{tabular}

Data are presented as median (interquartile range).

SCL-90R, Symptom Checklist-90-revised; FGIDs, functional gastrointestinal disorders.

Table 8. Multivariate Logistic Regression Analysis to Assess Psychological Factors Associated with Functional Gastrointestinal Disorders

\begin{tabular}{lccccc}
\hline \multicolumn{1}{c}{ Variable } & $\beta$ & Standard error & Wald & OR (95\% CI) & p-value \\
\hline Somatization & 0.132 & 0.026 & 26.518 & $1.141(1.09-1.20)$ & $<0.001$ \\
Obsessive compulsive & 0.081 & 0.026 & 9.316 & $1.084(1.03-1.14)$ & 0.002 \\
Depression & -0.059 & 0.025 & 5.574 & $0.943(0.90-0.99)$ & 0.020 \\
\hline
\end{tabular}

OR, odds ratio; CI, confidence interval.

disease category (5.5\% of total population). The most prevalent combination was FD with FH and FCP ( $n=7,3.9 \%$ of all FGIDs), followed by FD+FCP ( $n=6,3.3 \%$ of all FGIDs) and FD+IBS $(n=5$, $2.8 \%$ of all FGIDs). FD, FH, FCP, and IBS were the prevalent components of such combinations (Table 5).

\section{Psychological factors}

The total SCL-90R score, which indicates a global severity, was 24 (13 to 44$)$ in soldiers with FGIDs, which was significantly higher than 13 (5 to 28) found in soldiers without FGIDs (interquartile range). All nine categories of psychopathologic variables showed a higher score in soldiers with FGIDs than without FGIDs (Table 6).

In the analysis of overlapping syndromes, the total SCL-90R score showed a significantly higher score in soldiers with overlapping syndromes than in soldiers with single FGIDs (31 [18 to 57] vs 14 [5.75 to 29], p<0.001). The scores of all of the psychopathologic variables were also higher in soldiers with overlapping syndromes than in those with single FGIDs (Table 7).

Multivariate logistic regression analysis was performed to assess the associated psychological factors of FGIDs. The factors of somatization (odds ratio [OR], 1.141; 95\% CI, 1.09 to $1.20 ; \mathrm{p}<0.001$ ), obsessive compulsive behaviors (OR, 1.084; 95\% CI, 1.03 to $1.14 ; \mathrm{p}=0.002$ ), and depression (OR, 0.943; 95\% CI,
0.90 to $0.99 ; \mathrm{p}=0.020$ ) were found to be independent predictive factors for FGIDs (model chi-square test, $\mathrm{p}<0.001$; HosmerLemeshow's goodness-of-fit test, $\mathrm{p}=0.09$ ) (age, smoking, alcohol, BMI, and psychopathologic variables of SCL-90R adjusted) (Table 8).

\section{DISCUSSION}

FGIDs are prevalent in the general population. ${ }^{1}$ According to epidemiologic studies of IBS, which is the most widely investigated FGID, the prevalence of IBS is estimated to range from $3 \%$ to $27 \%$, considering differences in race and study methodology. ${ }^{11-19}$ A recent study in Korea found a prevalence of FGIDs in patients who visited primary clinics and tertiary care hospitals estimated at $49.7 \%{ }^{5}$ However, studies aiming at young populations are limited. Moreover, there are no data concerning FGIDs in the military.

This population consumes a similar diet and performs regular exercise with controlled life activities. The prevalence of total FGIDs in this population was estimated to be $18.5 \%$ in this study (age-adjusted prevalence, 18.1\%). This result is lower than that from a study of Chinese air force population (23.5\%). ${ }^{20}$ However, among the 4,170 soldiers, 1,070 officers with higher education and longer years of service were included in that 
study. Moreover, about 37\% of soldiers were aged over 25 (13.1\% were in age $>35)^{20}{ }^{20}$ According to the another study of Chinese college and university students of the young age group, the prevalence of total FGIDs was $23 \% .^{10}$ However, considering the fact that FGIDs are more prevalent in females, the results of our study may agree with those of the Chinese study. In a subgroup analysis of the Chinese study, the prevalence of FIGDs in males was $20.4 \%$, whereas that in females was $25.5 \% .^{10}$ Regarding the influence of the military, the strong military discipline instituted daily could cause serious stress, triggering FGIDs more easily than in the general population. ${ }^{21}$ However, regular diet and exercise, restricting alcohol and eating between meals could act as a positive impact on the development or progression of FGIDs. From a study on the impact of diet on FD, the regular diet group (three meals per day) showed a lower incidence of FD. ${ }^{22}$ There have been contradictory results about the relationship between obesity and FGIDs. ${ }^{23-25}$ In this study, few obese soldiers were enrolled, and the authors could not find differences in the BMI between soldiers with FGIDs and without FGIDs (Table 1). Overall, FGIDs are prevalent in the military; however, they are not more prevalent than in the general population of the similar age group, ${ }^{10}$ most likely due to the absence of factors contributing to the development of FGIDs.

In the subtype analysis of FGIDs, FD was the most prevalent disorder (8.2\%), followed by FCP (5.1\%), FH (2.8\%), IBS (2.6\%), and functional dysphagia (2.6\%) (Table 3). According to a Chinese study on the similar age group, FD showed the highest prevalence at 9.3\%, followed by IBS at 8.3\% and functional constipation at 5.5\%. ${ }^{10}$ However, this study estimated the prevalence of only three FGIDs, including FD, IBS, and functional constipation. The results of a hospital-based study in Korea were consistent with those of the Chinese study (FD, 46\%; IBS, $40.2 \%$ ), although the overall prevalence was much higher. ${ }^{5}$ In the present study and the Chinese study, no endoscopic investigation was performed to rule out organic diseases. Only alarm symptoms based the questionnaires were used. Thus, gastroesophageal reflux disease (GERD), which is not included in the Rome III criteria categories, could not be assessed. Regarding GERD patients in Korea, who commonly express symptoms similar to a burning sensation, a substantial portion of FCP and FH patients in this study might overlap with GERD. Cautious interpretations should be made regarding this aspect. The prevalence of IBS in this study was much lower than in the Chinese or Korean studies (2.6\%). ${ }^{5,10}$ The positive impact from dietary and daily activity factors could explain the lower prevalence. However, more studies are needed to identify the factors responsible for this result.

As shown in previous studies, a subtype of PDS was more prevalent than EPS among soldiers with FD ( $n=78$ vs 1 ). ${ }^{5,10}$ It has been speculated that the diagnostic criteria for EPS in the Rome III standard were too strict and that patients had difficulty understanding the meaning of the questionnaire with respect to differentiating between epigastric burning and heartburn. ${ }^{5}$ This problem is expected to be solved in the subsequent revised version of the Rome criteria. Of the subtypes of IBS, diarrhea type IBS was most prevalent $(n=10)$, followed by mixed $(n=9)$, constipation type $(n=3)$, and unsubtyped IBS $(n=3)$; however, the number of patients detected was too small to establish significant differences (Table 3).

In terms of overlapping syndromes, the prevalence was estimated at $5.5 \%$ of the total population and $29.6 \%$ of the FGID patients from this study. In previous studies, the prevalence has been reported to range from $1 \%$ to $17 \%$, not much different from our study. ${ }^{26-30}$ In a recent Korean study, the most common combination was FD+IBS, and the systematic review showed that the pooled OR of IBS in patients with FD was 8 (OR, 8; 95\% CI, 5.7 to 11.2). ${ }^{5,31}$ However, our study showed that FD+FH+FCP ( $n=7,3.9 \%$ of all FGIDs) was most prevalent, followed by FD+FCP ( $n=6,3.3 \%$ of all FGIDs) and FD+IBS ( $n=5,2.8 \%$ of all FGIDs) (Table 5). According to the study of healthy military males from United States, the most common combination of overlap syndrome was IBS+functional constipation. ${ }^{32}$ Functional constipation was also the most prevalent FGID in that study. ${ }^{32}$ The reason of the different combinations from studies are unclear. Another consideration is about GERD. FGIDs and GERD presumably share the same pathophysiology. ${ }^{19}$ However, the exact mechanism and prevalence of each combination is unclear. Regarding the pitfalls stated above in this study regarding GERD, some clinical aspects of GERD might be hidden by the results of this study (soldiers with FH or FCP might be included in GERD), although many other epidemiologic studies have also excluded GERD in the analysis of overlapping syndrome and FGIDs. According to one study on the overlap of GERD and FD, a prevalence of $4 \%$ was found in the general population. ${ }^{33}$ Overall, overlapping syndromes are commonly observed, not only in the general population but also in soldiers.

In agreement with the results of previous studies, psychological distress was associated with FGIDs and overlapping syndromes. ${ }^{5,10,22,28}$ All components of the SCL-90R showed a higher score in soldiers with FGIDs (than without FGIDs) and overlapping syndromes (than without overlapping syndromes) (Tables 6 and 7). In the multivariate logistic regression analysis to assess the associated psychological factors of FGIDs, the factors of somatization $(\mathrm{OR}, 1.141)$ and obsessive compulsive feature (OR, 1.084) proved to be independent risk factors for FGIDs. However, depression (OR, 0.943) revealed a negative relation to FGIDs (Table 8). In a Chinese study of the same age and race group, depression also showed an OR of 0.55, although the 95\% CI included 1.0 (95\% CI, 0.15 to $1.05 ; \mathrm{p}=0.045) .{ }^{20}$ According to a study of the brain-gut pathway, which is presumed to be one pathophysiology of the FGIDs, depression was not an independent predictor for developing FGIDs, whereas anxiety was a risk factor. ${ }^{34}$ The exact reason or mechanism is unclear regarding this issue. More studies are needed to elucidate the association 
between each of the psychological factors and FGIDs.

There are limitations to this study. First, this study used only self-administered questionnaires without endoscopic investigations. Secondly, the questionnaire used in this study was not validated. At the time of this study, the Korean version of the Rome III questionnaire, which was validated recently, was not available; however, it is currently applicable in clinical practice. $^{35}$

However, this study is the first epidemiologic study of FGIDs in the Korean military, and subsequent studies which have endoscopic investigation for all participants and survey with validated questionnaire can be planned and executed to better assess this population based on the results of this study.

In conclusion, FGIDs are common among men in their twenties completing military duty. Somatization and obsessive compulsive disorders deriving from the tense atmosphere are associated with the development or progression of FGIDs. Patients who have overlapping syndromes require greater attention due to their more severe psychopathology.

\section{CONFLICTS OF INTEREST}

The authors have declared that no competing interests exist. The funders had no role in study design, data collection and analysis, decision to publish, or preparation of the manuscript.

\section{REFERENCES}

1. Halder SL, Locke GR 3rd, Schleck CD, Zinsmeister AR, Melton LJ 3rd, Talley NJ. Natural history of functional gastrointestinal disorders: a 12-year longitudinal population-based study. Gastroenterology 2007;133:799-807.

2. Agarwal N, Spiegel BM. The effect of irritable bowel syndrome on health-related quality of life and health care expenditures. Gastroenterol Clin North Am 2011;40:11-19.

3. Jung HK. Rome III criteria for functional gastrointestinal disorders: is there a need for a better definition? J Neurogastroenterol Motil 2011;17:211-212.

4. Drossman DA, Dumitrascu DL. Rome III: new standard for functional gastrointestinal disorders. J Gastrointestin Liver Dis 2006;15:237-241.

5. Park JM, Choi MG, Cho YK, et al. Functional gastrointestinal disorders diagnosed by Rome III questionnaire in Korea. J Neurogastroenterol Motil 2011;17:279-286.

6. Kim ES, Lee BJ, Kim YS, Lee SI, Park H. Validation of Rome III criteria in the diagnosis of functional gastrointestinal disorders in Korean patients. Korean J Neurogastroenterol Motil 2008;14:3944.

7. Derogatis LR, Lipman RS, Covi L. SCL-90: an outpatient psychiatric rating scale--preliminary report. Psychopharmacol Bull 1973;9: 13-28.

8. Kim JH, Kim KI. The standardization study of Symptom Checklist- 90-revision in Korea III. Ment Health Res 1984;2:278-311.

9. Derogatis LR. SCL-90-R: Symptom Checklist-90-R: administration, scoring, and procedures manual. 3rd ed. Minneapolis: NCS Pearson, 1975.

10. Dong YY, Chen FX, Yu YB, et al. A school-based study with Rome III criteria on the prevalence of functional gastrointestinal disorders in Chinese college and university students. PLoS One 2013;8:e54183.

11. Talley NJ, Zinsmeister AR, Van Dyke C, Melton LJ 3rd. Epidemiology of colonic symptoms and the irritable bowel syndrome. Gastroenterology 1991;101:927-934.

12. Sandler RS. Epidemiology of irritable bowel syndrome in the United States. Gastroenterology 1990;99:409-415.

13. Drossman DA, Li Z, Andruzzi E, et al. U.S. householder survey of functional gastrointestinal disorders: prevalence, sociodemography, and health impact. Dig Dis Sci 1993;38:1569-1580.

14. Choung RS, Locke GR 3rd, Zinsmeister AR, Schleck CD, Talley NJ. Psychosocial distress and somatic symptoms in community subjects with irritable bowel syndrome: a psychological component is the rule. Am J Gastroenterol 2009;104:1772-1779.

15. American College of Gastroenterology Task Force on Irritable Bowel Syndrome, Brandt LJ, Chey WD, et al. An evidence-based position statement on the management of irritable bowel syndrome. Am J Gastroenterol 2009;104 Suppl 1:S1-S35.

16. Hahn BA, Saunders WB, Maier WC. Differences between individuals with self-reported irritable bowel syndrome (IBS) and IBS-like symptoms. Dig Dis Sci 1997;42:2585-2590.

17. Saito YA, Locke GR, Talley NJ, Zinsmeister AR, Fett SL, Melton LJ 3rd. A comparison of the Rome and Manning criteria for case identification in epidemiological investigations of irritable bowel syndrome. Am J Gastroenterol 2000;95:2816-2824.

18. Thompson WG, Irvine EJ, Pare P, Ferrazzi S, Rance L. Functional gastrointestinal disorders in Canada: first population-based survey using Rome II criteria with suggestions for improving the questionnaire. Dig Dis Sci 2002;47:225-235.

19. Choung RS. Natural history and overlap of functional gastrointestinal disorders. Korean J Gastroenterol 2012;60:345-348.

20. Wu W, Guo X, Yang Y, et al. The prevalence of functional gastrointestinal disorders in the chinese air force population. Gastroenterol Res Pract 2013;2013:497585.

21. Bennett EJ, Tennant CC, Piesse C, Badcock CA, Kellow JE. Level of chronic life stress predicts clinical outcome in irritable bowel syndrome. Gut 1998;43:256-261.

22. Mullan A, Kavanagh P, O'Mahony P, Joy T, Gleeson F, Gibney MJ. Food and nutrient intakes and eating patterns in functional and organic dyspepsia. Eur J Clin Nutr 1994;48:97-105.

23. van Oijen MG, Josemanders DF, Laheij RJ, van Rossum LG, Tan AC, Jansen JB. Gastrointestinal disorders and symptoms: does body mass index matter? Neth J Med 2006;64:45-49.

24. Delgado-Aros S, Locke GR 3rd, Camilleri M, et al. Obesity is associated with increased risk of gastrointestinal symptoms: a population-based study. Am J Gastroenterol 2004;99:1801-1806. 
25. Cremonini F, Locke GR 3rd, Schleck CD, Zinsmeister AR, Talley NJ. Relationship between upper gastrointestinal symptoms and changes in body weight in a population-based cohort. Neurogastroenterol Motil 2006;18:987-994.

26. Locke GR 3rd, Zinsmeister AR, Fett SL, Melton LJ 3rd, Talley NJ. Overlap of gastrointestinal symptom complexes in a US community. Neurogastroenterol Motil 2005;17:29-34.

27. Lee SY, Lee KJ, Kim SJ, Cho SW. Prevalence and risk factors for overlaps between gastroesophageal reflux disease, dyspepsia, and irritable bowel syndrome: a population-based study. Digestion 2009;79:196-201.

28. Kaji M, Fujiwara Y, Shiba M, et al. Prevalence of overlaps between GERD, FD and IBS and impact on health-related quality of life. J Gastroenterol Hepatol 2010;25:1151-1156.

29. Lee HJ, Lee SY, Kim JH, et al. Depressive mood and quality of life in functional gastrointestinal disorders: differences between functional dyspepsia, irritable bowel syndrome and overlap syndrome. Gen Hosp Psychiatry 2010;32:499-502.

30. Wang A, Liao X, Xiong L, et al. The clinical overlap between functional dyspepsia and irritable bowel syndrome based on Rome III criteria. BMC Gastroenterol 2008;8:43.
31. Ford AC, Marwaha A, Lim A, Moayyedi P. Systematic review and meta-analysis of the prevalence of irritable bowel syndrome in individuals with dyspepsia. Clin Gastroenterol Hepatol 2010;8:401409 .

32. Porter CK, Gormley R, Tribble DR, Cash BD, Riddle MS. The incidence and gastrointestinal infectious risk of functional gastrointestinal disorders in a healthy US adult population. Am J Gastroenterol 2011;106:130-138.

33. Choung RS, Locke GR 3rd, Schleck CD, Zinsmeister AR, Talley NJ. Overlap of dyspepsia and gastroesophageal reflux in the general population: one disease or distinct entities? Neurogastroenterol Motil 2012;24:229-234.

34. Koloski NA, Jones M, Kalantar J, Weltman M, Zaguirre J, Talley NJ. The brain: gut pathway in functional gastrointestinal disorders is bidirectional: a 12-year prospective population-based study. Gut 2012;61:1284-1290.

35. Song KH, Jung HK, Min BH, et al. Development and validation of the Korean Rome III questionnaire for diagnosis of functional gastrointestinal disorders. J Neurogastroenterol Motil 2013;19:509515. 